controlling for probes. The partial correlation coefficients are $0.5879,0.5418$ and 0.5482 for probes D4S139, D1S7 and D2S44, respectively. The partial correlation coefficients are $0.5557,0.5109$, $0.5072,0.8404$ and 0.5024 for G.S.R., A.M.G., L.A.W., H.L.L. and M.E.C., respectively. All the partial correlations have chance probabilities less than 0.001 .

In the control sample, the two bands are $a$ priori independent of each other. Before laboratory treatment, each band is just a constant from a constant sample. Nonetheless, it is clear from these results that the process by which the band length estimations are carried out destroys whatever independence exists between the two alleles of a pair of bands.

These findings have serious practical implications for the use of these databases to calculate population probabilities for forensic samples: (1) Bandlength estimates by different people may not be comparable. (2) The joint probability of obtaining a particular pair of band lengths within a sample is not equal to twice the product of the individual band-length probabilities. (3) A product rule for calculating probabilities across probes also fails because of the pattern of differences between technicians across probes. (4) The disproportional representation of technicians in the racial databases for each probe exacerbates these problems.

Department of Psychology,

MARTIN M. SHAPIRO

Emory University,

Atlanta,

Georgia 30322, USA

\title{
More natural classification
}

SIR - Woese et al. ${ }^{1}$, who criticised my proposal for the classification of the living world ${ }^{2}$, miss the basic issue. They fail to realize that there are two ways by which organisms can be ordered. One is the traditional system of classification used almost universally since Linnaeus by botanists and zoologists, in which organisms are grouped in classes on the basis of similarity and relationship. The other is Hennig's "general reference system", in which organisms are grouped strictly phylogenetically, that is according to what 'clade' they belong to ${ }^{3}$. The arrangement adopted by Woese et al. is Hennig's phylogenetic reference system; the classification supported by me is based on the traditional principles of classification, which biology shares with all fields in which items are classified, as are books in a library or goods in a warehouse.

Both groups of prokaryotes, the eubacteria and the archaebacteria, are very ancient and branched from each other before the origin of the eukaryotes. There is no argument between myself and Woese et al. as far as the facts are concerned. The argument is whether a hennigian reference system (containing only branching information) or a traditional classification is the more useful.

I opt for the latter for a number of reasons, some of which I have already stated $^{4}$. A hierarchy of categorical ranks in a traditional classification expresses degrees of difference. To give each of the two subdivisions of the prokaryotes the same rank (domain) as the eukaryotes, which differ by the possession of a nucleus, cytoplasmic organelles and many other drastic characteristics, violates all principles of hierarchial classification. Indeed the two groups of prokaryotes are so similar that the dis- tinctness of the archaebacteria was discovered by Woese as recently as 25 years ago. Archaebacteria and eubacteria are indeed so similar that the definition of bacteria found in the older textbooks of bacteriology fits both. I find it incomprehensible that Woese et al. can call a classification artificial which recognizes the drastic difference between prokaryotes and eukaryotes.

Woese $e t$ al. also violate another basic principle of classification, that the best scheme should be based on as many diagnostic differences as possible. Woese and other authors interested in the branching sequence have, however, limited themselves to a consideration of those few characters that had already branched among the prokaryotes. This ignores the vast majority of eukaryote characteristics and cannot help but result in an unbalanced consideration. A more detailed analysis of the many weaknesses of phylogenetic reference systems has been given elsewhere ${ }^{4}$.

Traditional classifications and hennigian phylogenetic reference systems have very different objectives and are, therefore, not alternatives. If one wants merely to document branching points in the phylogeny, one will use a hennigian cladogram. If one wants to express degrees of difference and more than merely linear relationship, one should adopt a traditional classification.

Museum of Comparative Zoology, ERNST MAYR

Harvard University,

Cambridge,

Massachusetts 02138, USA

1. Woese, C. R., Kandler, 0. \& Wheelis, M. I. Nature 351 , 528-229 (1991)

Mayr E. Nature 348, 491 (1990)

3. Hennig, W. Phylogenetic Systematics (University of Illinois Press, Urbana, 1966).

4. Mayr, E. \& Ashlock, P. D. Principles of Systematic Zoology (McGraw Hill, New York, 1991).

\section{Ice-age methane variations}

SIR - Chappellaz et al. ${ }^{1}$ note that methane fluctuations in the last glacial cycle, which have a strong 23,000-year (23-kyr) precession component ( $a$ in the figure), compare favourably with calculated precipitation variations ${ }^{2}$ in the area of the Asian monsoon (b). A further justification for the low-latitude origin was the observation that the highlatitude ice volume signal is dominated by 100 -kyr oscillations 3 .
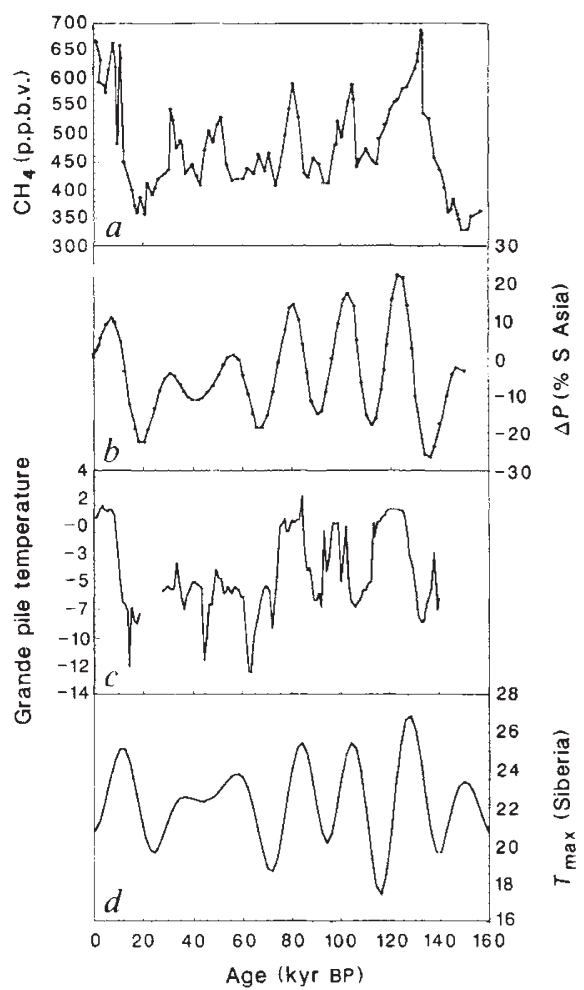

Comparison of methane ${ }^{1}$, southern Asian precipitation index ${ }^{2}$, a northeast France $\left(47^{\circ} \mathrm{N}\right)$ pollen-temperature record ${ }^{5,10}$, and central Eurasian $\left(60^{\circ} \mathrm{N}, 100^{\circ} \mathrm{E}\right)$ temperature index ${ }^{8}$. The last calculation used a twodimension energy balance model to examine the filtering effect of geography on orbital forcing. Previous comparisons of the model to general circulation models indicate that it has a sensitivity comparable to general circulation models for seasonal changes over land areas 11,12 .

Although 23-kyr fluctuations are commonly associated with low-latitude phenomenon, 23-kyr forcing also occurs in high latitudes (see, for example, ref. 4). A pollen record from northeast France $\left(47^{\circ} \mathrm{N}\right)$ has significant $23-\mathrm{kyr}$ power (refs 5,$6 ; c$ in the figure). Further east in Siberia, in the largely ice-free wetlands region that now stores a significant quantity of methane ${ }^{7}$, models of climate $^{8}$ indicate summer temperature variations at $60^{\circ} \mathrm{N}$ of about $106^{\circ} \mathrm{C}(d)$ that have a strong $23-\mathrm{kyr}$ component. In fact, the largest modelled temperature 